\title{
Gravitational Weakening of Seismic Origin as a Driving Mechanism of Some Astronomical Anomalies
}

\author{
Paul C. Rivera ${ }^{1}$ \\ ${ }^{1}$ Hymetocean Peers Co. Antipolo City, Philippines \\ Correspondence: Paul C. Rivera, 89 Almaciga St. Fin-Asia Subd. San Isidro Cainta 1900 Rizal, Philippines. \\ E-mail: paulcrivera2@gmail.com
}

Received: January 7, 2019

Accepted: January 25, 2019

Online Published: February 22, 2019

doi:10.5539/apr.v11n2p10

URL: http://dx.doi.org/10.5539/apr.v11n2p10

\begin{abstract}
The anomalous increase of the astronomical unit, the mysterious secular increase of the lunar eccentricity and the flyby anomaly are important issues of modern astronomy. This study aims to determine the effect of earthquakes on the origin of these anomalies. Based on conventional physics, we found the existence of earthquake-induced gravitational weakening within the earth-moon-sun system and beyond. New equations of gravitation, including time dilation, are introduced that can explain the anomalous increase of the astronomical unit and the lunar orbit. A modified equation that includes the effect of massive quakes also explains the anomalous increase of the lunar eccentricity. Furthermore, the results of the present study can explain the flyby and Pioneer anomalies experienced by spacecraft during gravity assisted maneuvers. A modification of the $3^{\text {rd }}$ Law of Kepler is also presented. Implications on the elliptic orbit of the earth, its reduced velocity and the occurrence of leap years are also discussed. Using the seismic-induced gravitational weakening model, probable trigger mechanisms of the faint young sun paradox and the Allais effect are also presented. An estimate of the age of the earth based on the observed values of the Hubble parameter and the USGS earthquake data for the past century is also presented. A new model of the seismic-driven expanding universe and a new equation to determine the expansion rate of galaxies and the universe is also proposed. The sudden earth's retreat due to gravitational weakening and its implication on anomalous astronomical refraction and flight risk at night especially near the equatorial region, and its effect on the abrupt satellite orbital decay, spin and drift are also discussed. This study may also shed light on the occurrence of sinkholes and massive landslides. Finally, this study proposes a new equation that can explain the observed changes in the fine structure constant.
\end{abstract}

Keywords: Gravitational Weakening, Massive Quakes, AU Anomaly, Flyby/Pioneer Anomaly, Time Dilation, Flight Risk, Satellite Orbital Decay, Gravity Anomaly, Galactic Rotation, Fine Structure Constant

\section{Introduction}

For the past century, astrophysicists have recorded several anomalies from actual observations of the earth-moon system and neighboring planets and their natural satellites. Observations show that some celestial objects, particularly the earth's moon, had been strangely moving away combined with an anomalous increase in the eccentricity of its elliptic orbit (Iorio, 2011). In addition, the astronomical unit (AU), which is the most accurately observed astrometric quantity, is also known to be anomalously increasing by a few $\mathrm{cm} /$ year (Krasinsky \& Brumberg, 2004; Anderson \& Nieto, 2010; Iorio, 2011; Miura et al., 2013; Acedo, 2014). Accordingly, the AU is increasing at about $7-15 \mathrm{~cm} /$ year. In addition, using historical eclipse records, the lunar orbit was confirmed to be increasing by about $2.82 \pm .08 \mathrm{~cm} / \mathrm{yr}$ (Riofrio, 2012). Previous studies also reported slightly larger values of about $4 \mathrm{~cm} / \mathrm{yr}$ (Stephenson, 1981). It was proposed that these astrophysical anomalies may have some cosmological origin or may call upon new physical driving mechanisms not taken into account by traditional Newtonian physics and General Relativity (Acedo, 2017).

Using conventional physics, this study attempts to elucidate the cause of these anomalies. A new physics as pointed out in Iorio (2014) and Acedo (2017) is not required since a previously neglected term that enters Newton's Second Law of motion has been found. This force which originates from the earth is the force associated with massive earthquakes. In general, it is a momentum transfer mechanism as celestial objects like the moon and earth, and artificial satellites are affected by geological forces not previously identified. 


\section{Theoretical Foundation and Extended Newtonian Gravitation}

Newton's law of universal gravitation is generally used to determine the gravitational force exerted by celestial objects like the earth-moon or earth-sun system, whose magnitude is given by;

$$
F=G \frac{M m}{R^{2}}
$$

where $G$ is the universal constant of gravitation, $M$ is the mass of the larger object, $m$ is the mass of the smaller object and $\boldsymbol{R}$ is the radius vector which represents the distance between the celestial objects. When one of the objects moves to a new distance $\boldsymbol{R}_{\boldsymbol{n}}$, Equation 1 becomes

$$
F_{n}=G \frac{M m}{R_{n}^{2}}
$$

This is possible as any transient perturbing force of sufficient magnitude can alter the orbital distance $\boldsymbol{R}$ to $\boldsymbol{R}_{\boldsymbol{n}}$. For instance, it was shown that the strong Sumatra quake of December 26, 2004 and its giant tsunami moved the moon by increasing the gravitational force resulting to the increased tilt of the planet earth as the moon receded (Rivera, 2011; Rivera \& Khan, 2012). Hence, transient gravitational weakening can occur due to massive quakes.

Differentiating Equation 1 with respect to time gives the equation of a gravitational jerk induced by a massive quake which is given by;

$$
\frac{d F}{d t}=-2 G \frac{M m}{R^{3}} \frac{d R}{d t}
$$

The relation $d \boldsymbol{F} / d t=m d^{3} \boldsymbol{R} / d t^{3}$ gives rise to gravitational jerks when used in this new equation. During a massive earthquake, the period of tremor is in the order of several seconds or a few minutes only. The episodic seismic effect is rather short and can be assumed as a small perturbation when compared with the period of orbital motion of the planet which is in the order of up to 1 year in the case of the earth around the sun. Therefore, we can eliminate the time derivative in Equation 3 to get

$$
d F=-2 \frac{F}{R} d R
$$

When $\boldsymbol{d} \boldsymbol{F}$ is integrated from $\boldsymbol{F}_{\boldsymbol{o}}$ to $\boldsymbol{F}_{\boldsymbol{n}}$ and the relative change of orbital distance $\frac{d R}{R}$ is integrated from $\boldsymbol{R}_{\boldsymbol{o}}$ to $\boldsymbol{R}_{\boldsymbol{n}}$, the analytical solution gives rise to a gravitational law that holds true for celestial objects that move away from their original orbits;

$$
F_{n}=F_{o}\left(1-2 \ln \frac{R_{n}}{R_{o}}\right)=G \frac{M m}{R_{o}^{2}}\left(1-2 \ln \frac{R_{n}}{R_{o}}\right)
$$

This holds for celestial objects affected by transient force mechanism due to massive quakes and may complement Newton's original law of universal gravitation. As the radial distance increases or decreases, the gravitational force must also decrease or increase. The force of the moon moving to and fro from its orbit as evidenced by super-moon occurrences and annular solar eclipses can be determined using this new gravitational equation.

\subsection{Seismic-Perturbed Gravitation - the Problem of Geo-Astronomical Jerks}

Unknown to many, the earth can perturb the moon in its orbit during massive quakes (Rivera, 2011). It is also possible that strong and long-lasting moonquakes can perturb the earth. As a result of the episodic oceanic force during earthquakes, the gravitational force between earth and moon may change with an associated change in the orbital distance between the earth and the moon (Rivera, 2011; Rivera \& Khan, 2012). Equation 5 can be rearranged to give

$$
R_{n}=R_{o} \exp \left(1-\frac{F_{n}}{F_{o}}\right) / 2
$$


However, dividing Equation 1 by Equation 2 also yields

$$
R_{n}=R_{o}\left(\frac{F_{o}}{F_{n}}\right)^{1 / 2}
$$

Equating 6 and 7 and rearranging gives

$$
F_{n}=F_{o}\left(1-\ln \frac{F_{o}}{F_{n}}\right)
$$

This simple non-linear equation is the basis of our gravitational weakening problem whose solution can be used to explain some of the observed astrometric anomalies in the solar system. Equations 7 and 8 represent a system of two simultaneous equations with two unknowns, namely; the gravitational force $\boldsymbol{F}_{\boldsymbol{n}}$ and the new orbital distance $\boldsymbol{R}_{\boldsymbol{n}}$ as a function of the known variables $\boldsymbol{F}_{\boldsymbol{o}}$ and $\boldsymbol{R}_{\boldsymbol{o}}$. The solution of Equation 8 gives rise to new astrophysical equations as shown below.

\subsection{Transcendental Function and Gravitational Implication}

The transcendental function given by Equation 8 can be reduced to a simple non-linear equation that can be solved using iterative methods. If we let the ratio $F_{n} / F_{o}=x$, Equation 8 can be written as $\boldsymbol{x}=\boldsymbol{1}+\boldsymbol{l n} \boldsymbol{x}$ or

$$
x=\exp (x-1)
$$

This equation can have a value of unity which implies no change in the gravitational force, $\boldsymbol{F}_{\boldsymbol{n}}, \boldsymbol{=} \boldsymbol{F}_{\boldsymbol{o}}$ and therefore $\boldsymbol{R}_{\boldsymbol{n}}=\boldsymbol{R}_{\boldsymbol{o}}$ according to Equation 7. This special case is true when the celestial object is not acted upon by any transient perturbing force and therefore remains in its original orbit. However, if the object has moved to a certain distance by virtue of an extra force which, in the case of the earth and moon is of geological origin, then the unknown parameter $\boldsymbol{x}$ must be less than unity. A value of less than unity indicates that the resulting force $\boldsymbol{F}_{\boldsymbol{n}}$ would have decreased from its original value $\boldsymbol{F}_{\boldsymbol{o}}$. Using an iterative solution technique known as the secant method (further explained below), the unknown quantity $\boldsymbol{x}$ was found to be exactly equal to 0.99999 . This value can be plugged in Equation 8 to confirm the identity. It is a correct solution which can also be verified by trial and error method. A short code in Basic Program (QB64) for the numerical solution is shown in the Appendix. Equation 8 is therefore reduced to

$$
F_{n}=9.9999 \times 10^{-1} F_{o}
$$

Subtracting $F_{o}$ from $F_{n}$ gives

$$
\Delta F=-1.0 \times 10^{-5} F_{o}
$$

This implies gravitational weakening since a small reduction in the gravitational force must occur during episodic seismic-induced jerks. Substituting Equation 10 in Equation 7 gives

$$
R_{n}=1.000005 R_{o}
$$

or

$$
\Delta R=5.0 \times 10^{-6} R_{o}
$$

These imply orbital expansion since a positive increase in the orbital distance is evident from Equations 12-13. This means that a celestial object like the earth undergoing a transient but strong tremor must recede and move farther from its original orbit. Thus, its orbit must expand and this is caused by the gravitational weakening shown by Equation 10. This can happen to the moon as well (and other seismically active planets and stars) since it is gravitationally bound to the planet earth. By virtue of episodic massive quakes, gravitational jerks could lead to orbital expansion and reduced force of attraction between celestial objects. Note that only one object needs to 
undergo episodic jerks to initiate expansion. This could be occurring in other astronomical objects as well. The large moon Titan on planet Saturn is found to be seismically active and might be a good candidate on the existence of gravitational weakening by massive quakes.

\section{Results and Discussion}

\subsection{Anomalous Increase of the Astronomical Unit}

The astronomical unit is the average distance between the earth and the sun. Recent astrophysical studies indicated that the average distance is increasing at a rate of about 7-15cm/year (Anderson \& Nieto, 2010; Iorio, 2011; Acedo, 2013). This rate of increase has not been fully understood and popular theories cannot explain its origin. In this study, we propose that this is caused by an astro-geological process - the self-propelled motion of the earth itself during massive quakes. To determine the anomalous rate of recession of the planet, we manipulate Equation 3 by applying Equation 11, noting that $d t \approx \Delta t$ as $\Delta t$ approaches 0 , to get

$$
\frac{d R}{d t}=\frac{5.0 \times 10^{-6} R}{\Delta t} \approx \frac{5.0 \times 10^{-6} R Q_{a}}{\Delta t}
$$

Here, $Q_{a}$ is the number of perturbing massive quakes. To get the right magnitude of the annual recession rate of the earth, the number of massive quakes per year must be multiplied with Equation 14 since there is not only 1 massive quake per revolution. Noting that the average $\mathrm{AU}(=\boldsymbol{R})$ is about $1.4959787 \times 10^{11} \mathrm{~m}$ and using Equations $12-13$, it can be seen that the AU should increase by about $+747,989.35 \mathrm{~m}$ (or $748 \mathrm{~km}$ ) with just 1 massive earthquake. This appears to be a large distance but not quite for a very massive planet floating in space and shaken by a massive tremor. While the earth orbits the sun in 1 year, quakes arise with a very short duration of a few seconds to minutes. The time duration $\Delta \mathrm{t}$ in the right hand side of Equation 14 is about $3600 \mathrm{sec} . \times 24$ hours/day $\times 365.25$ days per revolution. This must be used since the observed AU recession rate is made on an annual basis and not on a short duration seismic occurrence. Hence, the planet earth appears to move by about $0.0237 \mathrm{~m}$ per quake in each revolution. A massive quake is defined here as an earthquake with a magnitude $\geq$ M7.5 that is capable of perturbing the whole planet. The physical basis of this can be found in Rivera (2011) in which the total seismic-generated collision force $\boldsymbol{F}_{\boldsymbol{t}}$ is given by the sum of the hydrostatic pressure-force and the quake-generated lateral collision force, i.e.

$$
F_{t}=\rho\left[g+\frac{1}{4}\left(\frac{10^{M_{w}} g}{\rho(D+h) v}\right)^{1 / 3} N_{z}\right] L W h
$$

where $\rho$ is the seawater density, $\boldsymbol{g}$ is gravitational acceleration, $M_{w}$ is the moment magnitude of the earthquake, $D$ is the quake focal depth, $h$ is the seawater depth (m), $v$ is the kinematic viscosity of seawater, $N_{z}$ is the buoyancy frequency, $L$ is the length and $W$ is the width of disturbed oceanic area. The lateral collision velocity parameter between the seabed slope and the ocean column is given by the second term inside the parentheses. Using average oceanic parameters (i.e. $\rho=1025 \mathrm{~kg} / \mathrm{m}^{3}, \mathrm{~L}=1000 \mathrm{~km}, W=800 \mathrm{~km}, h=5 \mathrm{~km}, v=1.22 \times 10^{-6} \mathrm{~m}^{2} / \mathrm{s}$, a massive quake of $M_{w}=7.5$ can generate a force which is $\geq 20 \%$ of the earth-moon gravitational force. The seismic data of the United States Geological Survey (www.usgs.gov) show that there is an average of about 3.88 or 4 massive earthquakes per year since the year 1900. Multiplying this average number of massive quakes with the computed increase of $\mathrm{AU}$ in Equation 14 yields a recession rate of $9.48 \mathrm{~cm} /$ year $(0.0237 \mathrm{~m} \times 4 /$ year $)$. When the number of massive quakes in the past century till the present is considered (i.e. is $0.0237 \mathrm{~m} \times 3 /$ year or $6 /$ year), the resulting recession rate is about $7.11-14.22 \mathrm{~cm} /$ year which is well within the observed rate of increase of about $7-15 \mathrm{~cm} /$ year. Using the observed number of massive earthquakes per year from USGS, then the annual rate of increase of AU can be plotted as in Figure 1. As shown, there are years when the increase of AU is zero which means that the earth remained within the average AU since there were no massive quakes during those years. However, there are years (1938 and 2007) when the recession rate exceeded $20 \mathrm{~cm} /$ year due mainly to an increase in the number of massive quakes during those years. The average rate of recession is about $9.21 \mathrm{~cm} /$ year for the period considered. The dataset also showed that since 1980, there is an increasing trend in the recession rate. This is due mainly to the observed increase of massive quakes since then. 


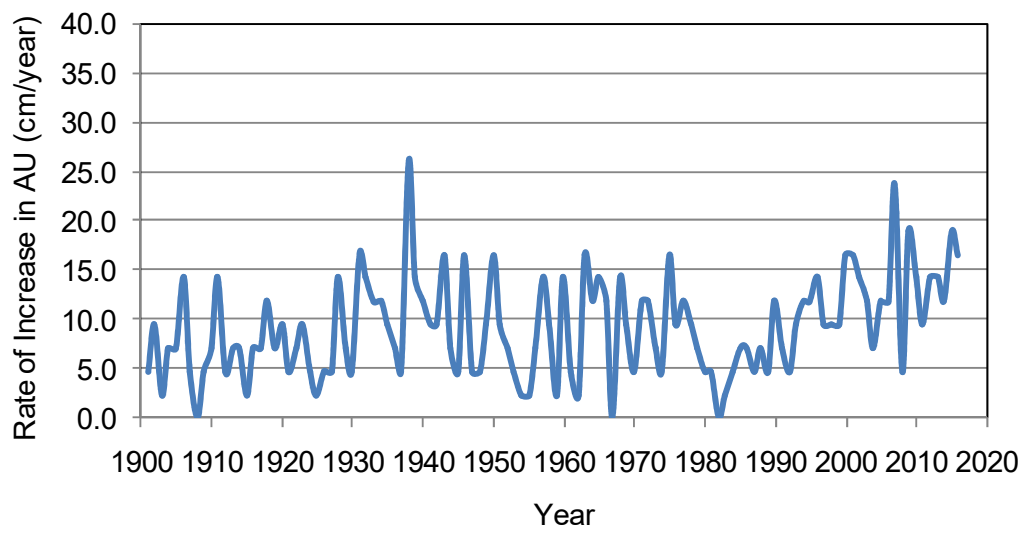

Figure 1. Estimated rate of increase of the Astronomical Unit (cm/year)

\subsection{Anomalous Recession of the Lunar Orbit}

The average lunar distance is about $384,400 \mathrm{~km}$ from the earth. Using Equations $12-13$, the orbital increase is about $1,922 \mathrm{~m}$ per massive quake. This is just about $6.09 \times 10^{-5} \mathrm{~m}$ in 1 earth year. Since the earth and moon are tidally locked and gravitationally bound, a massive earthquake should create a perturbation in the lunar orbit as well. This is due to the altered gravitational force between the earth and the moon during the quake. The Earthquake-Perturbed Obliquity Change Model (EPOCH) in Rivera (2011) and Rivera and Khan (2012) showed how earthquakes could alter the lunar position with a concomitant tilting of the earth as the moon moves farther. The collision force of the seismic-disturbed oceans of the earth appears to move the moon via a linear momentum transfer mechanism. The moon is less massive than the earth and naturally takes a weaker earthquake magnitude to agitate it. The USGS dataset since 1900 with magnitude $\mathrm{M}_{\mathrm{w}} \geq 6.0$ was used in this case since such quakes can already generate about $10 \%$ of the lunar force of attraction using the seismic-induced force model (Equation 15). Earthquakes with magnitude M6.0 is steadily rising since 1900 and in the year 2016-2017, there were 408-616 number of quakes recorded by USGS. Multiplying Equation 14 with this quake frequency, the rate of increase in the lunar orbit is about $2.48-3.75 \mathrm{~cm} /$ year. This estimate is close to the observed $2.82-3.83 \mathrm{~cm} /$ year reported in Riofrio (2012) and Williams et al. (2016).

Using the observed number of quakes with $\mathrm{M} \geq 6.0$, the annual variation of the change in lunar orbit is plotted in Figure 2. Note that the dataset for 1901-2013 was downloaded from USGS website in 2013 prior to the addition of other datasets. Data for 2014-2017 were downloaded only in 2018. The dataset has been extrapolated up to the year 2100 using an exponential fit for the quake count. An exponential funtion appears to give a good fit for the number of quakes until the year 2100 with $r=0.87$. From this, it can be seen that the rate of increase in the lunar orbit could double in less than 100 years due to seismic-induced gravitational weakening. However, this is still less than the oberved rate of increase in the AU. This is due to the fact that it is the earth that is perturbed during massive quakes and the moon just responds to the seismic-induced gravitational perturbation.

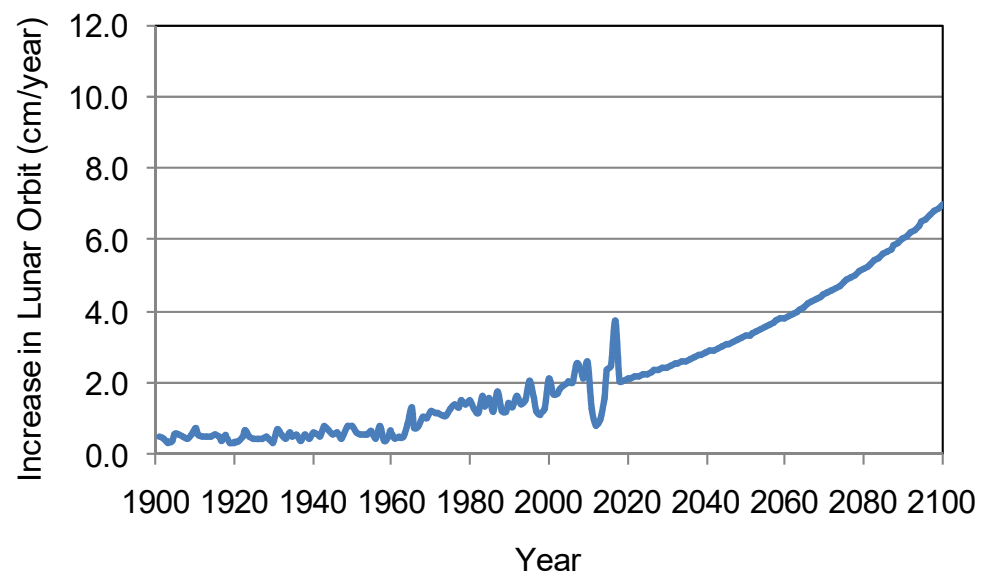

Figure 2. Computed and predicted rate of increase of the lunar orbit ( $\mathrm{cm} /$ year) 
It should be noted that as the moon recedes, the tide-generating potential which is proportional to $\mathbf{G M r} / \boldsymbol{R}^{3}$ where $\boldsymbol{R}$ is the mass of the earth and $\boldsymbol{r}$ is the radius, must decrease. This is also true for the tidal force whose magnitude is equal to $\mathbf{2 G M m r} / \boldsymbol{R}^{3}$. A $5 \%$ increase in $\boldsymbol{R}$ translates to over $13 \%$ reduction in the tidal force and tidal potential. The effect of massive earthquakes on the earth-moon distance provides a clear driving mechanism for the observed effect of tidal deceleration on earth and the receding moon. Massive quakes must have triggered the increase in the moon's orbit. Moreover, an increase in $\boldsymbol{R}$ increases the angular momentum and torque exerted by the moon on the earth and this also increases earth's obliquity as shown in Rivera (2011).

\subsection{Flyby Anomaly and the Pioneer Mystery}

Satellites in outer space normally undergo earth-assisted maneuvers to be able to reach their destinations. The astrodynamics of several spacecraft had become a mystery in the astrophysical community due to an unexplained loss or gain in energy and associated change in velocity during gravity assists. Some of these are listed in Anderson et al. (2005, 2007, and 2010) and Turyshev \& Toth (2009 and 2010). To determine the velocity change of the spacecraft during transient maneuvers, a balance between the magnitudes of the radial force and the gravitational force can be used;

$$
m_{s} \frac{V^{2}}{R}=G \frac{M m_{s}}{R^{2}}
$$

where $\boldsymbol{V}$ is the velocity of the spacecraft undergoing maneuver, $M$ is the mass of the earth, $m_{s}$ is the mass of the spacecraft and $\boldsymbol{R}$ is the radius vector from the earth's center. The mass $m_{s}$ and distance $\boldsymbol{R}$ cancel out and getting the derivative of both sides yields;

$$
d V=-\frac{G M d R}{2 V R^{2}}
$$

Substituting Equation 13 for $d \boldsymbol{R}$ by assuming that $d \boldsymbol{R}=\Delta \boldsymbol{R}$, gives

$$
d V=-5 \times 10^{-6} \frac{G M}{2 V R}
$$

This is the basis of the present flyby anomaly model. In satellite astrodynamics near the earth, the effect of the moon must be included in the analysis. To treat the earth-moon system as one body, a reduced mass is used in which $M=M m /(M+m)$ where $M$ is the mass of the earth and $m$ is the mass of the moon. To correct for the latitudinal position of the spacecraft, this should be divided by the cosine of the latitude $\Phi$ plus the average tilt (23.5 degrees) or obliquity of the earth, i.e. cos $(\Phi+23.5)$. It should be noted that the constant in Equation 18 is analogous to the constant $K\left(=3.099 \times 10^{-6}\right)$ derived by Anderson et al. (2007). However, there was no physical justification of the constant derived in the former study (Iorio, 2011). In this study, the constant was derived from the gravitational weakening and orbital expansion model (Equations 12-13) caused by massive quakes.

It should be noted that $\boldsymbol{R}$ is a radius vector and the negative sign in Equation 18 accounts for the fact that a spacecraft that approaches the earth must increase (negative $\boldsymbol{R}$ ) or decrease (positive $\boldsymbol{R}$ ) in velocity. Using Equation 18 in the data of Anderson et al. (2007), the newly estimated velocity anomalies are given in Table 1 below. Galileo 1 appears to be underestimated but only slightly, whereas Galileo 2 is accurately modeled. Cassini and Rosetta were also estimated accurately with same order of magnitude. The spacecraft NEAR appeared to be less than half the reported value. Nonetheless, this is still within the range of the observed magnitude of the velocity change. The signs are totally dependent on the motion of the spacecraft relative to the earth's center.

Table 1. Computed and observed flyby anomalies using the present model (Equation 18)

\begin{tabular}{llllll}
\hline Parameter & Galileo 1 & Galileo 2 & NEAR & Cassini & Rosetta \\
\hline Date & $12 / 8 / 90$ & $12 / 8 / 92$ & $1 / 23 / 98$ & $8 / 18 / 99$ & $3 / 4 / 05$ \\
Height $(\mathrm{m})$ & $9.600 \mathrm{E}+05$ & $3.030 \mathrm{E}+05$ & $5.390 \mathrm{E}+05$ & $1.175 \mathrm{E}+06$ & $1.956 \mathrm{E}+06$ \\
Speed $(\mathrm{m} / \mathrm{s})$ & $8.949 \mathrm{E}+03$ & $8.877 \mathrm{E}+03$ & $6.851 \mathrm{E}+03$ & $1.601 \mathrm{E}+04$ & $3.863 \mathrm{E}+03$ \\
Latitude (Degrees) & 25.20 & -33.80 & 33.00 & -23.50 & 20.20 \\
$\Delta$ V (observed), $\mathrm{mm} / \mathrm{s}$ & $\mathbf{3 . 9 2}$ & $\mathbf{4 . 6 0}$ & $\mathbf{1 3 . 4 6}$ & $\mathbf{- 2 . 0 0}$ & $\mathbf{1 . 8 0}$ \\
$\Delta$ V (computed), $\mathrm{mm} / \mathrm{s}$ & $\mathbf{2 . 1 4}$ & $\mathbf{- 4 . 5 7}$ & $\mathbf{5 . 9 4}$ & $\mathbf{- 0 . 6 4}$ & $\mathbf{2 . 2 2}$ \\
\hline
\end{tabular}


The new model presented here may disprove the hypothesis that the flyby anomalies were caused by collisions of the flybys with earth-bound dark matter (Adler, 2010).

It should be noted that the new flyby anomaly model given by Equation 18 can be used to explain the negative acceleration (directed towards the sun-earth system) of the Pioneer Spacecraft. In the case of Pioneer 10, we can use Equation 18 with $V=12.24 \mathrm{~km} / \mathrm{s}$ and $\boldsymbol{R}$ is about $21 \mathrm{AU}$ (start of unmodelled acceleration) with respect to Earth $\left(M=5.97219 \times 10^{24} \mathrm{~kg}\right), d V / d t$ within just a 30-second occurrence of massive quake yields $-8.63 \times 10^{-8} \mathrm{~cm} / \mathrm{s}^{2}$ which is very close to the observed deceleration of $-8.74 \times 10^{-8} \mathrm{~cm} / \mathrm{s}^{2}$ reported by Anderson et al. (2008). For Pioneer 11, recent studies also showed that Saturn's moon Titan is seismically active (Hayes et al., 2017). Assuming that Titan quakes occurred during the Pioneer 11 maneuver, then using $M=1.3452 \times 10^{23} \mathrm{~kg}$ for Titan, $V=11.6 \mathrm{~km} / \mathrm{s}$ and $\boldsymbol{R}=$ $80,000 \mathrm{~km}$ upon exit for Pioneer 11, then during the 8-hour anomalous period, the spacecraft acceleration according to Equation 18 yields $d V / d t=-8.39 \times 10^{-8} \mathrm{~cm} / \mathrm{s}^{2}$ which corresponds to the observed anomalous deceleration of about $-8.0 \times 10^{-8} \mathrm{~cm} / \mathrm{s}^{2}$. This is not trivial as the anomaly meant delayed arrival and that the Pioneer spacecraft was over $350,000 \mathrm{~km}$ short of their projected position. As the spacecraft left the Earth (and Saturn) to higher altitudes en-route to outer space, the velocities must have decreased due to massive quakes according to the new model leading to the observed anomalous deceleration. The new model shows that a conventional mechanism in the gravitation problem has been overlooked and is not an entirely new physics that is needed.

The implication of the new model on spacecraft approaching the earth with a hypersonic velocity must not be regarded lightly. The Columbia Space shuttle which disintegrated above the US territory in February 1, 2003 must have accelerated too fast upon entering the atmosphere resulting in extreme adiabatic heating and gas compression inside that might have resulted in the fatal accident of several astronauts and loss of the spacecraft. If the gravitational acceleration $\left(\mathrm{g}=9.8 \mathrm{~m} / \mathrm{s}^{2}\right)$ is added to the positive acceleration of the spacecraft during the approach given by Equation 18, there is a strong probability that this ignited the fire on the spacecraft as its acceleration exceeded the limit.

\subsection{Orbital Ellipticity and Anomalous Increase of Eccentricity}

Considering that the lunar recession is triggered by earthquakes, the anomalous eccentricity of the earth's orbit around the sun and the lunar orbit around the earth could be also due to occurrence of massive tremors. When the radial distance increases due to massive quakes, then the eccentricity $e$ of a planetary or lunar orbit around a host object can be estimated from

$$
e=Q_{a} \sqrt{1-\frac{R_{o}^{2}}{R_{n}^{2}}}
$$

This is similar to the conventional eccentricity formula but multiplied here by the number of massive quakes $\left(\boldsymbol{Q}_{a}\right)$ since they are found in this study to be responsible for gravitational jerks and orbital expansion. If there are no massive quakes, then $e=0$ which implies unperturbed circular orbit. The increased orbital distance due to massive quakes is $\boldsymbol{R}_{\boldsymbol{n}}\left(=1.000005 \boldsymbol{R}_{\boldsymbol{o}}\right)$ and this is equivalent to the semi-major-axis of an ellipse. Using this in Equation 19 with $\boldsymbol{Q}_{a}=5$ massive quakes, the resulting eccentricity of the earth's orbit is $\boldsymbol{e}=0.0158$. This is close to the observed eccentricity $\boldsymbol{e}=0.0167$. With 6 massive quakes in a year (i.e. $\boldsymbol{Q}_{\boldsymbol{a}}=6$ in 2017), the eccentricity increases to $\boldsymbol{e}=0.0189$. Hence, the eccentricity of the earth's orbit must have increased due mainly to seismic-induced weakening. This awaits further verification as more useful data become available. Equation 19 shows how effective massive quakes are in perturbing an otherwise circular orbit. The cause of the elliptic orbit was not clearly explained by Keplerian and Newtonian dynamics. If celestial objects (i.e. planets, moons or stars) outside of the solar system exhibit the same gravitational weakening due to quakes, then their elliptic orbits and periods are largely dictated by gravitational jerks from quakes. This shows that the eccentricity of planetary orbit is largely governed by self-propelled dynamic motion of the planet as episodic quakes perturbed its otherwise circular orbit. In the absence of a nearby perturbing object, the simple equation (19) can thus describe the anomalous elliptic orbits of celestial objects acted upon by massive quakes.

In the case of the moon, earthquakes with magnitudes of $M \geq 6.0-6.7$ can already perturb its orbit as shown by the seismic-generated force (Equation 15). Such quakes can already generate $\geq 10 \%$ of the lunar force of attraction. The average number of quakes with $M \geq 6.7$ is $Q_{a}=21$. Using this in Equation 19, then the lunar eccentricity $e=$ 0.0664 which is close to the observed eccentricity of 0.0647 . It should be noted that a quake of magnitude $M 6.7$ can generate only a slightly stronger oceanic force than a magnitude $M 6.0$ quake. Thus, the increasing frequency of earthquakes may govern the anomalous secular increase of the lunar eccentricity. This is a probable cause of the high lunar eccentricity, which has no theoretical explanation (Cuk, 2007). With increased number of massive 
earthquakes, the seismic-perturbed oceanic motion can displace the moon in its orbit. If the moon can drive oceanic tides, the strong motion of the oceans during massive quakes can also move the moon. The magnitude and direction of the oceanic force must govern the mysterious motion of the moon which puzzled many investigators including Newton and Einstein. Using Equation 15 with the giant quake and tsunami of 2004, the moon was thrown out of its orbit (i.e. $\boldsymbol{R}$ increased as shown by the small full moon) while during the massive Japan quake of March 2011, the moon was tugged by the earth where $\boldsymbol{R}$ decreased leading to the super-moon formation during that month.

\subsection{Gravitational Weakening Causes Length Expansion and Retards Orbital Velocity}

Gravitational weakening from massive earthquakes is shown to increase length as the orbital distance of the planet earth around the sun increases according to Equations 12-13. With several massive quakes per year, the distance of the planet earth in $n$ number of years of revolution should increase by

$$
R_{n}=R_{o}\left(1+\frac{\Delta R}{R_{o}} Q_{a} n\right)=R_{o}\left(1+5 \times 10^{-6} Q_{a} n\right)
$$

where $Q_{a}$ is the annual number of massive quakes, and $\boldsymbol{n}$ is the number of years of revolution. Note that Equation 20 reduces to Equation 12 for a single massive quake when $n=1$ year. Using Equation 20 with $n=100$ years and $Q_{a}=4$ quakes per year, the term in the parentheses equals 1.002 . Hence, the orbital distance of the earth will increase to about $1.4989737 \times 10^{11} \mathrm{~m}$ from the sun (almost $300,000 \mathrm{~km}$ ) in 100 years due mainly to massive quakes. Assuming a circular orbit, the present speed of orbital velocity of the earth is about $29,785.33 \mathrm{~m} / \mathrm{s}$. However, with massive quakes and associated gravitational jerks, the orbital velocity of the planet earth must also change according to;

$$
V_{n}=V_{o}\left(1+\frac{d V}{V_{o}}\right)^{n}
$$

where $\boldsymbol{V}_{\boldsymbol{n}}$ is the perturbed orbital velocity, $\boldsymbol{V}_{\boldsymbol{o}}$ is the initial orbital velocity and $n$ is number of years. The last term on the right is analogous to Equation 18 divided by velocity so that Equation 21 can be written as

$$
V_{n}=V_{o}\left(1-5 \times 10^{-6} \frac{G M_{\Theta}}{2 V_{o}^{2} R_{n}}\right)^{n}
$$

where $M_{\Theta}$ is the mass of the sun. This shows that the orbital speed of the planet must decrease during massive quakes. Using the computed change in the orbital distance, the term in the parenthesis is 0.999750684. Hence, the orbital velocity of the earth decreases to about $29,777.8999 \mathrm{~m} / \mathrm{s}$ in 100 years.

\subsection{Implication on the 3rd Law of Kepler and the Occurrence of Leap Years}

The average number of massive quakes at present is about 4 per year. Assuming that this was already the case in the past century (since USGS records started documenting since 1900 only, we do not know the average number), the reduced speed of the earth's revolution around the sun translates to an increase of about 0.82 day to the number of days in a solar year. This leads to the existence of a leap year with 1 additional day in every 4 years. It should be noted that the number of massive quakes determines this extra day. Thus, the $3^{\text {rd }}$ Law of Kepler on orbital periods has to be modified. Taking into account the increased orbit due to massive quakes (Equation 20), the law on orbital periods is now written as,

$$
T^{2}=\frac{4 \pi^{2}\left[R_{o}(1+k)\right]^{3}}{G M_{\Theta}}
$$

Here, $k=5 \times 10^{-6} Q_{a} n$ in which $Q_{a}$ is the number of massive quakes per $n$ revolution and $\boldsymbol{M}_{\boldsymbol{\Theta}}$ is the mass of the sun. For satellites and the moon revolving around the earth, the mass of the earth $M$ must be used in Equation (23) to get their mean motion. If there are no massive quakes, then the $3^{\text {rd }}$ Law of Kepler remains unchanged since $k=0$. The average number of massive quakes on earth at present is about 4, so the resulting solar year is now about 365.32211 days and not 365.24219 days. This amounts to 1 additional day almost every 3 years and not every 4 years. It can be stated therefore that the earth literally leaps in its orbit during the occurrence of massive quakes and 
slows down as it traces a larger orbit, leading to the leap year. The solar year has thus increased due to massive quakes and led to non-synchronized seasons (i.e. out of sync) with the present calendar. Looking back on the case of the advancement of 10 days in the Gregorian calendar in 1582 (e.g. October 4 was followed by October 15 in 1582 as ordered by then Pope Gregory XIII), it is highly probable that the resulting change is attributed to this phenomenon of reduced orbital speed of the earth as it orbits a longer distance. Since Year 0 to 1582, there was exactly a 13-day difference between the Gregorian calendar and the Solar Year. But the 3-day difference had been previously adjusted in the old Julian calendar so that there was a remaining 10-day difference that was removed. Apparently, even the solar year is not constant but changes depending on the number of massive quakes on earth. With the present rate of occurrence of massive quakes on earth, the Gregorian calendar may be corrected by having a leap year on February 29 every 3 years and not every 4 years.

\subsection{Gravitational Time Dilation Due to Massive Quakes}

When the gravitational weakening by massive quakes is projected to a number of years or centuries, the resulting reduction in the gravitational force between space objects like the earth and moon will not be trivial. This can be determined using the gravitational weakening equation given by

$$
F_{n}=F_{o}\left(1+\frac{\Delta F}{F_{o}}\right)=F_{o}\left(1-1 \times 10^{-5} Q_{a} n\right)
$$

where $\Delta F$ is given by Equation 11. The gravitational weakening given by Equation 10 is generalized here by incorporating the effect of $Q_{a}$ massive quakes in $n$ number of years. Within a 1,000 -year period therefore, the gravitational force would decrease by about $1 \%$. This is not a small amount since this is about $1.98 \times 10^{18} \mathrm{~N}$ of the gravitational force between earth and the moon without any additional mass or dark matter involved. It should be noted that earthquakes had been occurring since the world began because the earth is tectonically active. Hence, the resulting reduction in the gravitational force between the earth and moon if we assume a 10,000 year period is about $10 \%$ or $1.98 \times 10^{19} \mathrm{~N}$. Massive quakes, therefore, weakens the gravitational force between celestial objects. It should be noted that quakes may also be occurring in exo-planets and stars in other galaxies as shown by observations of stellar oscillations. Therefore, the gravitational weakening brought about by massive quakes is a highly probable physical mechanism of cosmic expansion. A cascade of gravitational weakening during conjunction or opposition of celestial objects in space may occur during strong tremors. The case of the moon being agitated by the earth during massive quakes has been shown in Rivera (2011) and Rivera \& Khan (2012). The anomalous advances of planetary perihelia reported in Iorio (2015) could also be due to seismic-induced gravitational weakening.

As gravity weakens, time is also affected since time $t$ equals distance $\boldsymbol{R}$ divided by speed $v$ (i.e. $t=\boldsymbol{R} / v$ ). Since the initial time $t_{o}=R_{o} / v$, then the ratio $t / t_{o}$, upon using Equation 20 for $\boldsymbol{R}$, yields

$$
t=t_{o}\left(1+5 \times 10^{-6} Q_{a} n\right)
$$

Thus, time must increase with the occurrence of massive quakes according to Equation 25. Without quakes, it is clear that time remains the same. There is therefore a positive increase of time $(\Delta t)$ for every massive quake that occurs. This is given by

$$
\Delta t=t-t_{o}=5 \times 10^{-6} Q_{a} n t_{o}
$$

This implies time dilation in which time increases forward (i.e. faster ageing) with more massive quakes. As gravitational force weakens during massive quakes, time apparently moves faster. This also means that the arrow of time can only move forward. The observed anomaly of astronomical time before massive earthquakes reported in $\mathrm{Hu}$ et al. 2016 and Wang et al. (2012) may be related to this phenomenon of seismic-induced gravitational time dilation.

\subsection{Implications on Dark Matter, Dark Energy and Age of the Earth}

When Equation 20 is used in Equation 5, there appears to be an extra mass causing an extra force tugging at celestial objects in space. But there is no hidden dark mass but only a change in the gravitational force caused by a change in the orbital distance. It only takes 22,500 massive quakes or 7,500 years with 3 massive quakes per year to produce an apparent dark matter of $27 \%$. Furthermore, there appears to be a repulsive force or dark 
energy in the universe as the gravitational force of celestial objects undergoing a jerk is reduced by virtue of Equations 10 and 24. But there is no repulsive force or dark energy. There is just a concomitant reduction in the gravitational force between celestial objects as the distance between them increases during massive quakes. It only takes 64,000 massive quakes or 16,000 years with 4 quakes per year to produce $68 \%$ apparent dark energy or repulsive force. The episodic jerks are largely responsible for the orbital expansion, changes in the gravitational force and apparent mass and energy increase. What appears to be a dark matter and dark energy can be explained by the new gravitational model. It is highly probable that there is no dark energy that causes the observed expansion of the universe and that the expansion is simply driven by massive quakes. The hypothesis that there are seismically active planets, moons and stars outside the solar system is becoming evident as space missions are currently getting useful seismic data. The recently concluded Cassini space mission indicates that Saturn's largest moon Titan is seismically active (Hayes et al., 2017). The apparent repulsive force is truly just a concomitant gravitational weakening as shown by the new models described above. When this occurs in other planetary and stellar systems in other galaxies in the cosmos, the resulting accelerated cosmic expansion could be the result of increased orbital distances during episodic jerks. If the rate of radial increase among seismic perturbed systems is mainly governed by seismically active planets, stars and moons in the solar system and other galaxies, then the observed rate of expansion based on the Hubble parameter can be used to estimate the age of the universe if we know the number of tremors in the whole universe. For the case of the earth, we consider the USGS data on earthquakes to estimate its current age. The Hubble parameter $H$ can be written as

$$
H=\frac{1}{R} \frac{d R}{d t}=\frac{5 \times 10^{-6} R Q_{a} n}{R \Delta t}
$$

Here, we use Equation 13 for $d \boldsymbol{R}$ and this cancels $\boldsymbol{R}$ so we can calculate the time period $\Delta t$ to deduce the present age of the earth based on observed Hubble data and USGS records. The observed Hubble parameter ranges from 160 to $67.3 \mathrm{Km} / \mathrm{s} / \mathrm{Mpc}$. When the lower limit is used for $H(67.3 \mathrm{Km} / \mathrm{s} / \mathrm{Mpc})$, and the average number of massive quakes for the period 1917-2017 ( $n=100$ years) is $Q_{a}=4$, then we obtain a time period $\Delta t=9.16992 \times 10^{14}$ seconds which translates to about 29.0577 million years. This is the proper age of the earth based on the observed values of the Hubble parameter and the number of massive tremors recorded by USGS in the past century. This is within the same order of magnitude obtained by various physicists and astronomers in the $19^{\text {th }}$ century like Helmholtz, Newcomb and Darwin using various techniques. This estimate appears short compared to the 4.54 billion years estimated by geologists. However, using the time dilation formula (Equations 25-26 with $\left.n=t_{o}\right)$, then $t=t_{o}+k t_{o}{ }^{2}$ where $k$ depends on the number of quakes and years. Assuming that $Q_{a}=1$ massive quake per year for the past 29 million years $\left(t_{o}\right)$, then the dilated and correct age of the earth is about 4.25 billion years according to our gravitational time dilation model. With $10 \%$ increase in massive quakes, this becomes higher (i.e. 4.64 billion years).

\subsection{Accelerating Expansion of the Universe}

When the Hubble equation (Equation 27) is integrated from $R_{o}$ to $R$, then a new model of the universe that expands in time due to massive tremors can be derived. Using a constant Hubble parameter $H_{o}$, on the left hand-side of Equation 27, and $Q_{t}$ as the average number of quakes in the universe, then we get an exponentially expanding universe according to

$$
R=R_{o} \exp \left[H_{o}\left(1+5 \times 10^{-6} Q_{t} n\right)\left(t-t_{o}\right)\right]
$$

Equation 28 means that the there is an accelerated expansion of the universe due largely to the occurrence of massive quakes. It also implies that the aging of the universe could have been accelerated due to the occurrence of gravitational weakening caused by episodic jerks. When there are no quakes (i.e. $Q_{t}=0$ ) in the universe, Equation 28 reduces to the exponential solution of the original Hubble formula. Note that it is possible to compute $R_{o}$ if we assume that the age of the universe is about 13 billion years and by assuming that $t_{o}=0$. But this requires the number of massive quakes in the cosmos which is not known. When estimates become available, for instance from galacto-seismology or astero-seismology, then we can readily determine the cosmic radial expansion at any time period.

The rate of expansion of the entire universe can also be estimated directly from Equation 14. At any radial distance $\boldsymbol{R}$, the rate of expansion for each year on earth, can be deduced simply by knowing the total number of quakes $Q_{t}$. The rate of expansion is proportional to $\boldsymbol{R}$ and $Q_{t}$ and is given by; 


$$
\frac{d R}{d t}=\frac{5 \times 10^{-6} R Q_{t}}{\Delta t}=1.584404391 \times 10^{-13} R Q_{t}
$$

For the planet earth, the computed and observed average expansion rate is about $9.48 \mathrm{~cm} /$ year using the observed $Q$ $=4$ massive quakes per year. However, for celestial objects located 100 light-years away, the expansion rate would be $d R / d t=150 Q_{t} \mathrm{~km} / \mathrm{year}$. Thus, assuming that in the cosmos there are 10,000 massive quakes annually, then the expansion rate of the universe would be $1.5 \times 10^{6} \mathrm{~km} /$ year. Equation 29 appears linear but not quite when Equation 20 is used for $R$. Hence, the expansion rate shows an accelerating tendency since the number of massive quakes $Q_{t}$ tends to accelerate with time. The USGS dataset alone shows an exponential increase of massive quakes $(\geq M 6.0)$ for the last century.

\subsection{Trigger Mechanism of the Faint Young Sun Paradox}

The seismic-driven orbital expansion model described above could also be the physical mechanism that explains the mystery of the faint young sun paradox. The solar radiation flux $S$ absorbed by the planets in the solar system varies inversely as the square of the radial distance from the sun as

$$
S=S_{o}\left(\frac{R_{o}}{R_{n}}\right)^{2}
$$

where $S_{o}$ is the solar constant. The evolution of the sun reveals that the solar output on earth was just about $75 \%$ of its present-day output (Iorio, 2015). However, the earth was warm enough to sustain life since liquid water was abundant throughout the planet. This paradox is difficult to explain as temperature all over the planet could have been much lower and waters including the oceans could have been frozen. However, the earth could also be much closer to the sun than it is at present time. It is highly probable then, that since the earth is tectonically active, massive quakes could have increased its radial distance from the sun as our new gravitational model shows (Equations 20 and 24). As the sun's output gradually increased, the distance between the earth and the sun slowly increased due to strong tremors. With a $75 \%$ solar output in the distant past, the distance between earth-sun could have been just $86.6 \%$ of the present $\mathrm{AU}$. This can be verified using Equation 30 with $R_{o}=R_{n}\left(S / S_{o}\right)^{1 / 2}$ which yields $R_{o}=1.295555 \times 10^{11} \mathrm{~m}$ when $\boldsymbol{R}_{n}$ equals the present $\mathrm{AU}\left(=1.495978707 \times 10^{11} \mathrm{~m}\right)$. Thus, $R_{o}$ could have gradually increased due to massive quakes to reach $\boldsymbol{R}_{\boldsymbol{n}}$. The proximity of the earth in the distant past with respect to the sun meant that the temperature of the planet was just enough to sustain liquid water and life. The elapsed time since the earth was at a distance $R_{o}$ to the present $\boldsymbol{R}$ can be estimated by using Equation 20. This gives $n\left(=t_{o}\right)$ of about $30,940.2$ years of revolution around the sun. Upon using the time dilation formulas (Equation 25-26), we obtain $t=$ $35,726.7$ years assuming 1 quake per year. Hence, with that quake frequency, it must have taken almost 36,000 years for the earth to reach its present distance from the sun.

\subsection{Most Probable Cause of the Allais Effect}

It was shown by the generalized gravitational model presented above that the earth moves away from the sun during massive quakes with a net effect that the gravitational force between the earth-moon system and the sun is altered. The gravity vector has a component that points towards the moon/sun during a solar eclipse but a massive quake causes earth's gravity to increase in the opposite direction. Thus, this should cause a change in the gravitational acceleration that we observe on earth and a change in a pendulum's period of oscillation during a solar eclipse. The possible driving mechanism of this effect is the disruption of the oceanic waters during the earthquake. During a submarine quake, a strong oceanic force is generated by the displaced oceanic mass with a net acceleration and force components that are either directed towards the moon or the earth (Equation 15). A lateral collision force is generated as the continental slope and the oceans collide during a massive quake (Rivera, 2006; Song et al., 2008) and this can move the moon from its orbit through the complementary action of the perturbed hydrostatic force of the ocean. The giant quake in the Indian Ocean in December 2004 for instance, caused a westward oceanic acceleration with a component directed towards the moon (Rivera, 2011). In contrast, the Japan quake and tsunami of March 2011 caused a strong eastward oceanic acceleration that moved the moon closer to earth (i.e. a super-moon occurred after the Japan quake). The displaced oceanic mass during massive quakes usually moves in an area larger than the area of disturbed submarine fault/trench as shown by observations and tsunami models (Rivera, 2006). This leads to both the perturbed motion of the earth and enhanced tilting of the planet which can cause a pendulum to swing faster and tilt. Following McCulloch (2008), the relative change of the period of the pendulum can be determined from; 


$$
\frac{d T}{T}=\sqrt{\frac{m_{o}}{M}}
$$

where $m_{o}$ is the displaced oceanic mass and $M$ is the mass of the earth. Using average values of displaced oceanic water during a massive quake of $M 7.5\left(m_{o}=750 \mathrm{~km} \times 400 \mathrm{~km} \times 5 \mathrm{~km}\right.$ mean depth $\left.\times 1025 \mathrm{~kg} / \mathrm{m}^{3} \mathrm{density}\right)$, the displaced oceanic mass may reach $1.5375 \times 10^{18} \mathrm{~kg}$. Note that the area of disturbed ocean will give the quake magnitude, i.e. $2 / 3 \log (L W)$ with $\mathrm{L}=750,000 \mathrm{~m}$ and $\mathrm{W}=400,000 \mathrm{~m}$. Plugging the values of mass, we obtain a relative change in the period of about 0.000507 which is close to the observed value of 0.0005 during an eclipse (Duif, 2004; Saxl \& Allen, 1970). Thus, the Allais effect may not be due to dark matter or inertial mass but simply due to displaced oceanic mass as quakes rock the earth. The absence of the Allais effect in some instances could be due to the intermittent occurrence of quakes and that the temporal disruption of the oceans by massive quakes will not last very long as the oceanic waters act to dampen massive quakes.

\subsection{Origin of the Anomalous Refraction}

For over a century, astronomers had been puzzled by the constant displacement of celestial objects like stars when ground-based telescopes are used to observe them. A number of studies had been conducted to determine the effect of the atmosphere on the cause of the problem. Previously, it was believed that the anomaly is caused by temperature and pressure gradients in the atmosphere or the passage of atmospheric gravity waves or tilted air mass layers. However, Taylor (2009) and Taylor et al. (2013) showed that gravity waves could not cause the anomalous refraction and the culprit is most likely due to a coherent dynamic motion below $60-\mathrm{m}$ in the planetary boundary layer. We postulated that this could be also due to seismic-induced planetary motion as the earth retreats due to many quakes. Using the computed motion of the earth during massive quakes (Section 3.1), there is a tremendous and sudden retreat of the earth as gravity weakens (i.e. about $748 \mathrm{~km}$ per quake). If this is divided by the number of seconds and hours in a day and multiplied by the number of night hours of observation, we obtain an apparent dynamic motion in the lower $60-\mathrm{m}$ of the atmosphere. When the earth recedes from the sun, the positions of ground-based telescopes must also retreat. This could lead to the observed anomalous refraction and apparent light bending (or gravitational lensing) as the recession is proportional to the zenith distance. The anomalous refraction $\varepsilon$ could be due to the motion of the whole planet during massive quakes and can be determined using the conventional refraction $(=k \tan z)$ formula and Equation 14 as

$$
\varepsilon=k_{r} \tan \Delta R=k_{r} \tan \left(5 \times 10^{-6} R Q_{a} n\right)
$$

Here, $\Delta R$ is the change in the earth's distance from the sun (proportional to zenith distance $z$ ) as the earth recedes, $k_{r}$ is the refraction coefficient which depends on atmospheric conditions, $Q_{a}$ and $n$ are the same as above.To obtain the independent contribution of earth's retreat, we assume that the effect of atmospheric condition is constant and the refraction coefficient is constant $\left(k_{r}=4 / 3-1\right)$. The argument inside the parentheses must be converted to degrees and radians by assuming that 1 mile equals 1 minute. With $n=1$ year and $Q_{a}=2$ massive quakes per year, then $\varepsilon=0.0924$ ". With increasing number of massive quakes $\left(Q_{a}=4\right.$ to 5 massive quakes per year), the anomalous refraction increases to $\varepsilon=0.2002$ " and 0.2674 ", respectively These estimates are within the range of 0.1 " to 0.3 " anomalous refraction reported in various studies (Hirt, 2006; Taylor, 2009). The number of massive quakes taken from the USGS data showed that there were 11 in 1938 and 7 in 1975. This yields higher refraction anomalies when used in Equation 32. Thisanomalous refraction is a critical problem in astrometry, air traffic control, radar systems, and ballistic missiles since targetsappear to move away from their true position just because the position of ground-based radar or telescopesareaffected by seismic-induced motion.This has serious implications in technologies using continuous radar observations.

\subsection{Potential Cause of Aerial Accidents and Clear Air Turbulence}

The aerial accidents of Air France in 2009, AirAsia Flight in 2014, and the missing Malaysian Airlines of 2014 may be related to the seismic-induced gravitational weakening and retreat of the earth with respect to the sun. As the gravitational force of the earth-sun systemweakens due to a repulsive action from quakes, this forces the earth to recede from the sun making aviation dangerous at night especially near the equatorial region. This is because a sudden retreat of the earth leaves the plane suddenly positioned much lower than its altitude. A $748-\mathrm{km}$ retreat in 15 minutes means that the plane appears to suddenly drop from the sky at $-2,992 \mathrm{~km} / \mathrm{hr}$, leaving the pilot or co-pilot confused and the plane's auto-pilot sytem to sound alarm. Even if the pilot attempts to pull down the gear (i.e plane goes up and does not stall), the plane would be overtaken by the sudden retreat of the earth and will appear to stall 
or plunge at sea. The upward speed of the plane is just too slow when compared to the recession speed of the planet and its oceans which amounts to over 2 times the speed of sound.

In addition, the sudden retreat of the earth away from the sun can cause clear-air turbulunce at daytime since strong air currents would be generated as the atmosphere and the land/oceans below the plane suddenlymoves down. Updraft and downdraft air speeds exceeding typhoon wind conditions in a large area can be expected due the sudden motion of the earth. Even with a planetary recession speed of $748 \mathrm{~km}$ in 1 hour, abruptly ascending air masses with vertical velocities of $207 \mathrm{~m} / \mathrm{s}$ along the path of the plane would cause very strong turbulence in the absence of any convective system or thunderstorm.

\subsection{Abrupt Orbital Decay, Spin and Drift of Satellites}

There is an unexplained abrupt satellite orbital decay which cannot be attributed solely to atmospheric drag because of very low level of air molecules in the upper atmosphere. Low earth orbit (LEO) satellites had been crashing to earth much earlier than predicted as the orbital decay becomes very fast. Many satellites have been continually observed by the project CASTOR (Canadian Automated Small Telescope for Orbital Research) and Earl \& Wade (2015) also found that box-winged satellites undergo episodic spins that last up to $1548 \mathrm{sec}$ (25.8min). In addition, there is an observed satellite drift of less than 1 degree per year. All of these could be due to earth's retreat caused by seismic-induced gravitational weakening that perturbs the earth and the satellites from their original orbits. Once the earth retreats due to massive quakes, torques around satellites should be generated and cause the satellites to spin. Moreover, they also appear to move downwards (or upwards) depending on their positions during earth's recession. The induced torque should cause satellites to spin within some seconds to several minutes. In addition, satellites would appear to drift from their initial orbits as the planet moves. This hampers satellites in their observational missions and their position, navigation and timing could yield dubious results. Precise orbit determination should take this into consideration. The effect of massive quakes on the orbital motion of satellites can be inferred from the vis-viva Equation given by:

$$
v=\sqrt{G M\left(\frac{2}{R_{o}}-\frac{1}{R_{n}}\right)}
$$

where $v$ is the velocity of the satellite in orbit. When Equation 20 is used for $\boldsymbol{R}_{\boldsymbol{n}}$ (i.e. the semi-major axis), the resulting vis-viva equation becomes

$$
v=\sqrt{\frac{G M}{R_{o}}\left(\frac{1+2 k}{1+k}\right)}
$$

where $k=5 \times 10^{-6} Q_{a} n$. It is evident from Equation 34 that the orbital velocity of satellites increases with massive quakes by a factor $\left(1+\frac{k}{k+1}\right)$. Without quakes $\left(Q_{a}=0, k=0\right), v$ reduces to the velocity around a circular orbit with radius $\boldsymbol{R}_{\boldsymbol{o}}$. Thus, episodic jerks largely govern the deviation of satellite motion from a classical circular orbit defined by Newton and Kepler. The living force as defined by the vis-viva equation appears to originate from earthquakes. The generalized vis-viva equation can also be derived directly from the Virial Theorem on kinetic energy where the gravitational force (Equation 1) is multiplied by $\boldsymbol{R}$ from Equation 20.

Hence, all satellites orbiting the earth at various altitudes would increase in velocity as the number of earthquakes increase. Then with enhanced orbital velocity, satellites would descend abruptly which is a source of big error in orbit determination and satellite measurements if not accounted for. The quake magnitude responsible for this could be less than M6.0. A cm-precision of satellite measurement in ocean altimetry and land topography is just difficult to achieve with this physical problem. In addition, precise orbit determination for satellites with a $\mathrm{mm}$-precision is too ambitious to achieve with the newly discovered phenomenon of seismic-induced gravitational weakening. On the other hand, this phenomenon can help engineers design a new spacecraft that can navigate through inter-stellar medium.

\subsection{Implication on Galactic Rotational Curve and Elliptical Galaxies}

The rotation curve of galaxies shows that the radial velocity increases with increasing distance from the galactic center. Confirmed by WMAP observations and visible images, this is paradoxical since Newtonian dynamics showed that velocity should be inversely proportional to radial distance $R$. However, we can derive a new equation for galactic rotation by assuming that the centripetal force is balanced by the radiation pressure gradient force instead of the Newtonian gravitational force as in; 


$$
\frac{V^{2}}{R}=-\frac{1}{\rho} \frac{\partial P}{\partial R}
$$

The radiation pressure $P$ is given by $P=P_{o}-4 \sigma T^{4} / c$ where $\rho$ is baryonic density, $P_{o}$ is a constant radiation pressure, $c$ is the speed of light, $\sigma$ is the Stefan-Boltzmann constant and $T$ is temperature. Differentiating this with respect to $R$ and substituting the result in Equation (35) gives;

$$
V=4 \sqrt{\frac{\sigma T^{3} R}{\rho c} \frac{\partial T}{\partial R}}
$$

Even in the absence of massive black holes, this new model holds true for rotating galaxies. We can also derive a similar equation using the luminosity $L=4 \pi r^{2} \sigma T^{4}$ (Chaisson \& McMillan, 2001) in the radiation pressure $P=P_{o}$ $L / \pi c r^{2}$ where $r$ is the stellar radius. It is easy to show that this yields $V=\sqrt{\frac{R}{\pi \rho c r^{2}} \frac{\partial L}{\partial R}}$. The baryonic density $\rho$, which has been observed to be unnaturally big (Kirilova, 2018), must be used here. When the temperature or luminosity profiles are accurately determined from observations, these new models should be able to describe galactic rotation curves. The spin velocity now depends on the square root of $R$, which can easily fit the observed rotation curves. With increasing distances, the velocity curve may level out or slightly decrease with actual gradients of temperature or luminosity. Assuming now that the galaxy of interest has star-quakes, then the perturbed radial distance must increase accordingly, and the galactic rotational speed (Equation 36) must also take into consideration the increase in $\boldsymbol{R}$ by using Equation 20. To obtain a good fit between computed and observed velocities, the emerging field of galacto-astero-seismology must consider also the number of galactic quakes. The chaotic motion of stars in various galaxies could be a manifestation of massive quakes especially in elliptical galaxies where the eccentricity of orbital motion is also governed by Equation 19. The more quakes occur in galaxies, the more elliptic should be their orbits as predicted by the new model. Moreover, they must age faster as shown by the gravitational time dilation formula presented above. This could be manifested by less luminous stars in elliptical galaxies.

\subsection{Alternative Cause of Continental Plate Movement, Sinkholes and Massive Landslide}

The estimated recession rate of the moon due to massive quakes using Equation (14) is about $3.75 \mathrm{~cm} /$ year. This is within the same magnitude as the observed rate of continental drift and plate movement $(1.3-10 \mathrm{~cm} /$ year). If we divide the distance between South America and South Africa (about 7,700km) by this mean recession rate of the moon, we get the amount of time needed for the continents to split apart (ca. 205 million years). Hence, the orbital changes of the moon and earth system and the associated changes in the gravitational force between the two due to earthquakes may cause continental/plate motion. Using Equation 10, a single massive quake can produce a $\Delta \boldsymbol{F}=2 \times 10^{15} \mathrm{~N}$ of gravitational force which is enough to split continents. By using Equation 3, the change in the gravitational force exerted by the earth's action on the moon and vice-versa ranges from $7.7 \times 10^{17}$ $4.6 \times 10^{18} \mathrm{~N} /$ year with $d R / d t=0.0237-0.1422 \mathrm{~m} /$ year (computed using Equation 14). The erratic motion of the moon due to massive earthquakes may further enhance the upwelling of volcanic magma from the earth which is presently accepted to be the chief cause of plate tectonic motion through convection currents.

The occurrence of sinkholes and massive landslides at present could also be due to gravitational anomaly caused by the expanding orbit of the earth. The potential change or anomaly in the gravitational acceleration is given by the derivative of $g=G M / r^{2}$, where $r$ and $M$ are the radius and mass of the earth;

$$
d g=-2 \frac{G M}{r^{3}} d r ; \Delta g=-10^{-5} \frac{G M}{r^{2}}
$$

where $d \boldsymbol{r}=\Delta \boldsymbol{r}=5 \times 10^{-6} \boldsymbol{r}$ (Equation 13). The earth does not expand, but the radial distance apparentlyincreases as the earth recedes due to gravitational weakening. This is especially true near the equatorial region or the ecliptic plane. For 1-6 massive quakes, Equation 37 yields a gravitational anomaly $\Delta g= \pm 9.79$ to \pm 58.7 mgals which are within the same magnitude measured by the GRACE satellite. This becomes a strong tangential force on the earth's surface particularly in mountain slopes when a large area or volume is soaked by torrential rains leading to collapse of the unstable surface.

As the earth moves away from the sun, it slows down causing an imbalance between the radial force and the gravitational force (i.e. $\boldsymbol{v}^{2} / \boldsymbol{R}<\boldsymbol{g}$ ). The self-gravitation from the planet becomes larger leading to an increase in the value of gravitational acceleration as the planet recedes. Any unstable area will collapse by virtue of $\Delta \boldsymbol{g}$ since the 
surface area times depth and enhanced density of wet materials leads to an enormous mass that makes the tangential force so great as to exceed the resisting force of the slope.

As self-gravitation of the planet now exceeds centripetal force, sinkholes may also occur as the inward force could overcome any resisting force leading to the collapse of earth materials even in plain surfaces. As $\Delta \boldsymbol{g}$ is higher in higher latitudes due to the oblate ellipsoidal surface of the earth, sinkholes may readily occur in high latitudes as well.

\subsection{Probable Cause of the Observed Lunar Ice: Momentum-Energy Conveyor Hypothesis}

The presence of frozen hydrogen and water ice on the lunar poles as shown by Li et al. (2018) may prove to be coming from the earth's oceans during tsunamis. It is possible that energy from submarine quakes is transferred from the oceans to the moon through a momentum-energy conveyor by hydrogen mechanism and not by a hypothetical graviton. The oceans comprise great amount of hydrogen water as $\mathrm{H}_{2} \mathrm{O}$ molecules, and the outer space with an enormous amount of hydrogen ions. While directed towards the west of the Indian Ocean, the giant Asian tsunami of December 2004 had a force component (i.e. force is rate of momentum change) that was directed towards the moon. The collision of oceanic hydrogen particles with hydrogen atoms in space during the tsunami could have imparted a considerable momentum and energy to the moon which drove the moon farther from its orbit. Hydrogen is an energy carrier and must be able to impart momentum and mass as well including hydrogen and oxygen molecules. Terada et al. (2017) also observed that the moon is contaminated with an oxygen isotope that can only be found on earth. Hence, any change in the earth's momentum during massive submarine quakes must drive a change in the lunar momentum through the law of linear momentum conservation, i.e. $M V=m v$ where $m$ and $v$ refer to the moon's mass and velocity, respectively. The earth's mass $M$ is constant but the collision speed $\boldsymbol{V}(=d \boldsymbol{R} / d t)$ is not and is proportional to the seismic-induced earth's recession rate given by Equation 14 . Considering the average masses of earth and moon, the mean earth-moon distance of $384,400 \mathrm{~km}$, and the present average of 6 massive earthquakes per year, then the lunar recession speed is $v=2.96 \mathrm{~cm} / \mathrm{year}$. This is also close to the $2.82 \mathrm{~cm} /$ year estimated in Riofrio (2012) and the estimates given above.

The linear momentum transfer mechanism is also evident in the observed anomalous precession of the planet mercury. The sun erupts with a hydrogenic coronal mass ejection $m=3.8 \times 10^{12} \mathrm{~kg}$ with a mean velocity $\boldsymbol{v}=$ $489,000 \mathrm{~m} / \mathrm{s}$. The energy and momentum carried by the enormous mass of hydrogen gas during massive solar eruption is manifested by a change in the momentum of the planet mercury. By using the law of linear momentum conservation, $m v=M V$ with the mass of the planet mercury $M=3.285 \times 10^{23} \mathrm{~kg}$, then the recession velocity of mercury is $V=5.629 \times 10^{-6} \mathrm{~m} / \mathrm{s}$ or $177.63773 \mathrm{~m} /$ year $\times 100 / 30.87=575.4 \mathrm{arcsecond} /$ century. This is the same magnitude as the presently observed advance of mercury perihelion which is about 575 arcsecond/century.

\subsection{Mechanics of the Hydrogen Momentum Transfer in Space}

The quantum aspect of the hydrogen atoms on earth and outer space can also be used to explain the linear momentum transfer mechanism considered here. There is an enormous amount of di-hydrogen atoms in seawater since water covers about $71 \%$ of the earth's surface. The de Broglie wavelength of hydrogen atoms is about $2.3 \times$ $10^{-10} \mathrm{~m}$ and this is greater than the diameter of the hydrogen atom which is about twice the Bohr radius or $1.058 \times$ $10^{-10} \mathrm{~m}$. Hence, the hydrogen atom transport mechanism can be considered quantum mechanically. However, there appears to be a tremendous amount of hydrogen atoms involved in the momentum transfer that the stream of hydrogen proton must be considered simultaneously.

The total seawater mass perturbed by the giant Asian tsunami of 2004 was about $1,200 \mathrm{~km} \times 550 \mathrm{~km} \times 4 \mathrm{~km} \times$ $1025 \mathrm{~kg} / \mathrm{m}^{3}$ or $2.71 \times 10^{18} \mathrm{~kg}$. The total hydrogen mass involved in the seawater mobilized by the massive quake was about 0.1119 times the seawater or $\mathrm{m}=3.028 \times 10^{17} \mathrm{~kg}$. Considering the excess energy of the hydrogen atom which is about 7,288.969 KeV, its velocity $v$ must be equal to $3.7 \times 10^{7} \mathrm{~m} / \mathrm{s}$, upon using the kinetic energy formula $\left(T=m_{p} \boldsymbol{v}^{2} / 2\right)$ and the mass of the hydrogen proton $m_{p}$. The product of the mobilized hydrogen mass and this velocity is the hydrogen momentum $p=m v$ which is equivalent to $1.122 \times 10^{25} \mathrm{~kg}-\mathrm{m} / \mathrm{s}$. On the other hand, the lunar momentum as it orbits the earth is $M V=7.519 \times 10^{25} \mathrm{~kg}-\mathrm{m} / \mathrm{s}$. Hence, the hydrogen momentum was about $15 \%$ of the lunar momentum and this is capable to disrupt the lunar orbit. This is the same magnitude as the ratio of the oceanic force $\left(3 \times 10^{19} \mathrm{~N}\right)$ exerted by the giant tsunami and the lunar gravitational force $\left(2 \times 10^{20} \mathrm{~N}\right)$ obtained in Rivera \& Khan (2012). The kinetic energy $T\left(=p^{2} / 2 m\right)$ carried by the hydrogen mass is about $2.08 \times 10^{32} \mathrm{~J}$. This enormous energy can be conveyed or transferred to the moon by the hydrogen ions during massive quakes.

A possible explanation of the momentum-energy-mass conveyor hypothesis by proton transfer can be invoked from the 200-year old Grotthuss mechanism or hydrogen hopping process. As the submarine quake perturbed the earth's oceans, the shock heating excited the hydrogen atoms in the seawater, and this then mobilized the hydrogen ions abundant in space. In the process of momentum and energy transfer, transport of mass particularly of oxygen molecules could occur as well and so the lunar pole has frozen $\mathrm{H}_{2} \mathrm{O}$ molecules ( $\mathrm{Li}$ et al., 2018). Hence, mass, 
energy and momentum could be readily conveyed from the earth to the moon during massive quakes. The momentum-energy transfer by hydrogen ions should be present in other seismically perturbed celestial objects as well since hydrogen atoms dominate the baryonic mass of the universe. Proton conductivity might play a crucial role in the momentum-mass-energy transport mechanism in other galaxies in outer space.

\subsection{Massive Quakes and the Fine-Structure Constant}

The massive quakes occurring on earth and other celestial objects like stars in distant galaxies may explain the observed small variations in the fine-structure constant introduced by Sommerfeld almost a century ago. Using first principles and the possible effect of massive quakes discussed above, the fine structure constant can be obtained from the ratio of the binding energy of hydrogen atoms and the proton to electron mass ratio $\mu$ given by

$$
\alpha=\frac{13.6(1+10 Q)}{n_{e}^{2} \mu}
$$

where $\mu(=1836.15)$ is the ratio of the hydrogen proton and the hydrogen electron, $Q$ is the number of quakes, and $n_{e}$ is the energy level or quantum state of the hydrogen electron. Here it is assumed that without quakes $(Q=0)$, the hydrogen electron remains in the ground state $\left(n_{e}=1\right)$ and Equation 38 gives the approximate value of the fine structure constant. As massive quakes occur, hydrogen atoms are excited through various mechanisms including shock heating and fluorescence by ultraviolet or ultralow frequency (ULF) radiation from the earth. As reported in Itoh et al. (2003), Herbst et al. (1996) and Thi et al. (1999) showed that these are the two mechanisms for hydrogen emissions in stars. Shock heating and release of ULF radiation could be occurring on earth during massive quakes as well. It is probable that during quakes, the hydrogen electrons are excited to higher energy levels. As the electrons decay to the lowest energy level, they emit ultraviolet light to form the Lyman Series (Ahmadi et al., 2018). The ULF radiation, with wavelengths of about $280 \mathrm{~nm}$, has the right energy magnitude that can ionize hydrogen atoms in space. The analyses made by Murphy et al. (2002) and Reinhold et al. (2001) have shown that the fine-structure constant $\alpha$ and $\mu$ are slightly changing. To determine the magnitude of these changes, we get the derivative of Equation 38 by assuming that only $\alpha$ and $\mu$ vary in time, and dividing by $\alpha$ to yield

$$
\frac{d \alpha}{\alpha}=\frac{-13.6(1+10 Q)}{\alpha n_{e}^{2} \mu} \frac{d \mu}{\mu}
$$

This shows that the relative change in the fine-structure constant $\frac{\Delta \alpha}{\alpha}$ is proportional to the relative change in the proton to electron mass ratio $\frac{\Delta \mu}{\mu}$, as their finite differences approach 0 . Evidently, this can be achieved when the electrons are excited to higher energy levels as quakes occur (with an apparent change in the hydrogen proton to electron mass ratio). Measurements made by Murphy et al. (2008) and King et al. (2008) showed that $\frac{\Delta \mu}{\mu}$ ranged from $1.8 \times 10^{-6}$ to $2.6 \times 10^{-6}$. Using the former value in our model, then with $n_{e}=12$ and $Q=11$, Equation 39 gives $-1.41 \times 10^{-6}$ which is close the most recent value $-1.42 \pm 0.55 \times 10^{-6}$ obtained by Kotus et al. (2016). Equation 39 may have a correction factor of $\cos (\Psi)$ where $\Psi$ is referenced to the ecliptic or galactic plane. Thus, it appears that the fine structure constant decreases with increasing seismic activities. With frequent quakes, the ionized hydrogen atoms convey momentum-energy-mass from perturbed objects to outer space possibly through instantaneous proton conductivity. The presently observed small variations of the fine-structure constant are caused mainly by seismic activities in the universe. Star-quakes in distant galaxies may be manifested by stellar oscillations similar to massive coronal ejections from the sun. In the process, it is expected that seismically active objects would lose hydrogen mass. The earth loses 95,000 tons of hydrogen per year probably due to increased seismic activities. The sun also loses massive hydrogen mass during Coronal Mass Ejection (CME) since every CME is a massive tremor in the sun. It is estimated that over $3.6 \times 10^{12} \mathrm{~kg}$ of hydrogen mass could be emitted per massive CME. This is also the most probable explanation in the reduction of the mass parameter $\left(G M_{\Theta}\right)$ of the sun. Chaotic motion of stars in every direction could be due to episodic tremors causing gravitational weakening and radial expansion. Consequently, the value of the fine structure constant in outer space is changing in every direction. Small changes in the fine-structure constant could be a manifestation of the changes in the mass-energy and momentum carried by hydrogen protons throughout space and consequently, the total hydrogen mass which is the most abundant element in the universe.

\section{Conclusions and Recommendations}


In this study, the existence of earthquake-induced gravitational weakening exhibited by seismically perturbed objects like the earth-moon system has been found. Based on conventional physics and using a simple algorithm, this study produces new gravitational equations related to Newtonian gravitation that can explain the cause of observed astronomical anomalies including the AU and Flyby/Pioneer anomalies. The study further shows how the earth's eccentricity and the secular increase of the lunar eccentricity are affected by gravitational weakening caused by massive tremors on earth. The new orbital expansion model could also explain the orbital velocity reduction of the earth and the occurrence of leap years. The $3^{\text {rd }}$ law of Kepler on orbital periods is also modified. Using the new gravitational model, a probable explanation of the Allais effect and the faint young sun paradox is also presented by taking into consideration the quake-driven earth's motion.

The anomalous perihelion precession of Saturn can also be explained using the new orbital expansion model. For instance, using Equations 14 and 20 with the mean distance between Saturn and its moon Titan as $\boldsymbol{R}=1,221,850 \mathrm{~km}$, then with 10 massive quakes per year, $d R / d t=0.0019359 \mathrm{~m} /$ year or $0.00627^{\prime \prime} \mathrm{cy}^{-1}$ which is close to the observed anomalous precession of Saturn $\left(0.006 \pm 0.002^{\prime \prime} \mathrm{cy}^{-1}\right)$ computed by Pitjeva (2008) as reported in Iorio (2015). However, the number of massive quakes in Titan should be verified in future space missions. Saturn itself or its other moons may also be prone to quake occurrence. The precession of Uranus could also be due to quakes in either Uranus or its moon Mab. With a mean distance of $614,092.44 \mathrm{~km}$ and assuming 12 massive quakes per year, the distance between Mab and Uranus increases at about 3.78 mas cy $^{-1}$ (close to the observed 3.89 mas cy $^{-1}$ reported in Iorio (2015).

The escape velocity $v_{e}$ of a spacecraft leaving the earth may also affected by the occurrence of massive quakes;

$$
v_{e}=\sqrt{\frac{2 G M}{r}}
$$

where $r$ is the radial distance from the earth's center (i.e. $6,378 \mathrm{~km}$ radius $+748 \mathrm{~km}$ earth's retreat per massive quake). The distance $r$ must incorporate orbital expansion using Equation 20. The earth does not expand but the orbital distance does expand due to massive quakes. Thus, $v_{e}$ must also decrease. For each massive quake, the decrease in the escape velocity of a spacecraft leaving near the equator can be estimated more accurately by incorporating orbital expansion due to quakes. The maximum height of the spacecraft must also be altered.

The results of this study shed light on the non-existence of dark matter and dark energy (or dark gravity) surrounding the earth-moon system and beyond. Evidently, there is an orbital expansion and associated gravitational weakening due to massive quakes and this causes an apparent repulsive force and not by a dark energy. The newly discovered dwarf galaxy NGC 1052-DF2 (van Dokkum et al., 2018) which is devoid of 'dark matter' might be due to the absence of gravitational weakening from massive quakes. On the other hand, elliptical galaxies could also point to the existence of seismic-induced gravitational weakening due to the occurrence of massive quakes and their ellipticity could have been enhanced by massive jerks.

The new astronomical model presented here can be used to determine the accelerated expansion of the universe. The expansion rate is largely driven by massive quakes and a new equation is proposed to determine the expansion of galaxies and the universe in general. In addition, a new estimate for the age of the earth using the time new dilation formula, the observed values of the Hubble parameter and the USGS quake data for the past century is shown to agree with geological estimates. The study may also explain the origin and small changes of the cosmological fine structure constant. It now appears that hydrogen is the medium responsible for gravitation and that changes in the momentum and energy carried by ionized hydrogen atoms define how gravity works and why gravity is weak. This renders our new gravitation theory compatible with quantum mechanical principles.

Other astrophysical problems will also be affected by the new orbital expansion model described here including the angular momentum, torque, the work done by perturbed celestial objects, and the kinetic energy exerted by seismically displaced objects including satellites. The observed different recession velocities of quasars may also be due to episodic jerks and can be estimated using Equation 29.

\section{Acknowledgments}

I wish to acknowledge the help of Marco Immanuel B. Rivera (National Institute of Physics -University of the Philippines) who assisted in writing the equations. Long discussions with him on many physical problems in the oceans, outer space and corresponding astrophysical mechanisms greatly helped in the completion of the study.

\section{Conflicts of Interest}

The author declares no conflict of interest. The author was fully responsible in the design of the study; in the collection, analyses, or interpretation of data; in the writing of the manuscript, and in the decision to publish the results. 


\section{References}

Acedo, L. R. (2013). Anomalous post-Newtonian terms and the secular increase of the astronomical unit. Adv. Space Res., 52, 1297-1303.

Acedo, L. R. (2015, July). The Flyby Anomaly in an Extended Whitehead's Theory. Galaxies, 3, 113-128.

Acedo, L. R. (2017, November). Anomalous accelerations in spacecraft flybys of the Earth. ArXiv e-prints.

Adler, S. L. (2010). Modeling the flyby anomalies with dark matter scattering. International Journal of Modern Physics A, 25, 4577-4588.

Ahmadi et al. (2018). Observations of the 1S-2P Lyman- $\alpha$ transition in antihydrogen. Nature.

Anderson et al. (2005, March). Study of the anomalous acceleration of Pioneer 10 and 11. ArXiv e-prints.

Anderson, J. D., \& Nieto, M. M. (2010, January). Astrometric solar-system anomalies. In S. A. Klioner, P. K. Seidelmann, \& M. H. Soffel (Eds.), Relativity in Fundamental Astronomy: Dynamics, Reference Frames, and Data Analysis (Vol. 261, pp. 189-197).

Anderson, J. D., Campbell, J. K., \& Nieto, M. M. (2007). The energy transfer process in planetary flybys. New Astron., 12, 383-397.

Chaisson, E., \& McMillan, S. (2001). Astronomy: A beginners guide to the universe (3rd ed.). Prentice-Hall, Inc. New Jersey, USA.

Cuk, M. (2007). Excitation of Lunar Eccentricity by Planetary Resonances. Science, 318(5848), 244.

Duif, C. P. (2004). A review of conventional explanations of anomalous observations during solar eclipses. ArXiv e-prints, 2004.

Earl, M. A., \& Wade, G. A. (2015). Observations of the Spin Period Variations of Inactive Box-wing Geosynchronous Satellites. J. Spacecraft \& Rockets, 52(3), 968-977.

Hayes, A. G., Birch, S. P. D., Dietrich, W. E., Howard, A. D., ..., \& Wood, A. (2017). Topographic Constraints on the Evolution and Connectivity of Titan's Lacustrine Basins. J. Geophys. Res., 2017.

Hirt. (2006). Monitoring and analysis of anomalous refraction using a digital zenith camera system. Astronomy and Astrophysics, 459(1).

Hu, H., Su, Y. J., \& Gao, Y. F. (2016). Anomalous astronomical time-latitude residuals: A potential earthquake precursor. Appl. Geophys, 13, 570.

Iorio, L. (2008). On the recently determined anomalous perihelion precession of Saturn. ArXiv e-prints Dec. 2008.

Iorio, L. (2011). An Empirical Explanation of the Anomalous Increases in the Astronomical Unit and the Lunar Eccentricity. The Astronomical Journal, 142(68).

Iorio, L. (2011). On the anomalous secular increase in eccentricity of the orbit of the Moon. Mon Not. Royal Astr. Soc., 415, 1266-1275.

Iorio, L. (2015). Gravitational Anomalies in the Solar System? International Journal of Modern Physics D, 24, 1530015-343.

Ito, Y., Sugitani, K., Ogura, K., \& Tamura, M. (2003). Detection of Molecular Hydrogen Emission Associated with LkHa 264. Publ. Astron. Soc. Japan, 55, L77-L81.

King, J. A., Webb, J. K., Murphy, M. T., \& Carswell, R. F. (2008). Stringent null constraint on cosmological evolution of the proton-to-electron mass ratio. Arxiv Preprints.

Kirilova, D. P. (2018). Non-equilibrium Processes in the Early Universe: Cosmological Constraints. Book Review. Bulg. Astron. J.

Kotus, S., Murphy, M., \& Carswell, R. (2017). High-precision limit on variation in the fine-structure constant from a single quasar absorption system. MNRAS, 464(3), 3679-3703.

Krasinsky, G. A., \& Brumberg, V. A. (2004). Secular Increase of Astronomical Unit from Analysis of the Major Planet Motions and its Interpretation. Celestial Mech. Dyn. Astron 2004, 90, 267-288.

Li, S., Lucey, P. G., Milliken, R. E., Hayne, P. O., ..., \& Elphic, R. C. (2018). Direct evidence of surface exposed water ice in the lunar polar regions. PNAS, 115(36), 8907-8912.

McCulloch, M. E. (2008). Modeling the flyby anomalies using a modification of inertia. MNRAS, 389, L57-L60. 
McCulloch, M. E. (2013). Can the flyby anomalies be explained by a modification of inertia? ArXiv e-prints.

Miura, T., Arakida, H., Kasai, M., \& Kurumata, S. (2013). Secular increase of the Astronomical Unit: A possible explanation in terms of the total angular momentum conservation law. Publ. Astro. Soc. Japan, 1.

Murphy, M., Flambaum, V., Muller, S., \& Henkel, C. (2008). Strong Limit on a Variable Proton-to-Electron Mass Ratio from Molecules in the Distant Universe. Science, 320, 1611-3.

Murphy, M., Webb, J., Flambaum, V., \& Curran, S. (2002). Time evolution of the fine structure constant. Arxiv e-Prints.

Pitjeva, E. V. (2008). Ephemerides EPM2008: The updated models, constants, data. Paper presented at Journ'ees "Syst'emes de r'ef"erencespatio-temporels" and X Lohrmann-Kolloquium (pp. 22-24). Dresden, Germany.

Reinhold, E., Buning, R., Hollenstein, U., Ivanchik, A., Petitjean, P., \& Ubachs, W. (2006). Indication of a Cosmological Variation of the Proton-Electron Mass Ratio Based on Laboratory Measurement and Reanalysis of H2 Spectra.

Riofrio, L. (2012). Calculation of lunar orbit anomaly. Planetary Science, 1.

Rivera, P. C. (2006). Modeling the Asian Tsunami Evolution and Propagation with a new generation mechanism and a nonlinear dispersive wave model. Sci. Tsunami Hazards, 25(1), 18-33.

Rivera, P. C. (2011). Discovery of the major mechanism of global warming and climate change. Proceedings of $G C G W$. Lisbon, Portugal. Retrieved from http://www.cge.uevora.pt/GCGW/presentations/105-15-1-RV.pdf

Rivera, P. C., \& Khan, T. M. A. (2012). Discovery of the major mechanism of global warming and climate change. J. Bas. Appl. Sci., 8(1), 59-73.

Saxl, E. J., \& Allen, M. (1971). 1970 Solar Eclipse as seen by a torsion pendulum. Phys. Rev. D, 3, 823.

Song, Y. T. (2007). Detecting tsunami genesis and scales directly from coastal GPS stations, Geophys. Res. Lett., 34, L19602.

Song, Y. T., Fu, L. L., Zlotnicki, V., Ji, C., Hjorleifsdottir, V., Shum, C. K., \& Yi, Y. (2008). The role of horizontal impulses of the faulting continental slope in generating the 26 December 2004 tsunami. Ocean Mod., 20, 362-379.

Stephenson, F. R. (1981). Tidal recession of the moon from ancient and modern data. J. Brit. Astron. Assoc., 91(2), 136-147.

Taylor, M. S. (2009). On the sources of astrometric anomalous refraction. PhD Dissertation. University of New Mexico. USA.

Taylor, M. Z., McGraw, J. T., Zimmer, P., \& Pier, J. R. (2013). On the source of astrometric anomalous refraction. Astro. Journal, 145(3), 82

Terada, K., Yokota, S., Saito, Y., Kitamura, N., Asamura, K., \& Nishino, M. N. (2017). Biogenic oxygen from Earth transported to the Moon by a wind of magnetospheric ions. Nature Astron, 1(0026).

Turyshev, S. G., \& Toth, V. T. (2009). The Puzzle of the Flyby Anomaly. Space Sci. Rev., 148, 169-174.

Turyshev, S. G., \& Toth, V. T. (2010). The Pioneer Anomaly. Living Reviews in Relativity.

Van Dokkum, P., Danieli, S., Cohen, Y., Merritt, A., ..., \& Zhang, J. (2018). A galaxy lacking dark matter. Nature, 555, 629-632. Retrieved from http://www.usgs.gov. Available online

Wang, B., Yin, Z. Q., \& Han, Y. B. (2012). Progress of research on anomalies of astronomical time and latitude observations before earthquake. Chin. Sci. Bull., 57, 3547-3555.

Webb, J., Murphy, M., Flambaum, V., Dzuba, V., ..., \& Wolfe, A. (2001). Further Evidence for Cosmological Evolution of the Fine Structure Constant. Phys. Rev. Lett., 87, 091301.

Williams, J. D., Boggs, D. H., \& Ratcliff, J. T. (2016). Lunar Tidal Recession. 47th Lunar and Planetary Science Conference.

\section{Appendix}

\section{Numerical Solution using Secant Method}

The transcendental function (Equation 9) can be represented by $f(x)=x-e^{(x-1)}$. With the secant method, any change in the numerical approximation must be equal to 


$$
\Delta \mathrm{x}_{\mathrm{i}}=\frac{f(x)_{i}}{f(x)_{i}-f(x)_{i-1}}\left(x_{i}-x_{i-1}\right)
$$

The new value of the unknown variable $\boldsymbol{x}_{\boldsymbol{i}}$ is then updated from

$$
x_{i+1}=x_{i}-\Delta x_{i}
$$

The secant method uses two initial values. With initial guess of $x(1)=0.1$ and $x(2)=1.0$, the numerical solution easily converges after 25 iterations as shown in Table A1. A short Basic (QB64) code to solve the mathematical problem is written below. It can be seen that the numerical solution converges very fast after 25 iterations with a negligible error or convergence criterion of $1 \times 10^{-10}$.

\section{PROGRAM SOLVEX.BAS}

$\mathrm{ni}=100:$ epsilon $=1.0 \mathrm{e}-10$

$\operatorname{dim} x(n i+1), f x(n i), d x(n i)$

$x(1)=0.1: x(2)=1.0$

for $\mathrm{i}=1$ to $\mathrm{ni}$

if $\mathrm{i}=1$ then $\mathrm{fx}(1)=\exp (\mathrm{x}(1)-1)-\mathrm{x}(1)$ else $\mathrm{fx}(\mathrm{i})=\exp (\mathrm{x}(\mathrm{i})-1)-\mathrm{x}(\mathrm{i})$

$\mathrm{dx}(\mathrm{i})=\mathrm{fx}(\mathrm{i}) /(\mathrm{fx}(\mathrm{i})-\mathrm{fx}(\mathrm{i}-1)) *(\mathrm{x}(\mathrm{i})-\mathrm{x}(\mathrm{i}-1))$

$x(i+1)=x(i)-d x(i)$

print $\mathrm{i}, \mathrm{x}(\mathrm{i}), \mathrm{fx}(\mathrm{i})$

if $\mathrm{fx}(\mathrm{i})<$ epsilon goto 100

next

100 end

Table A1. Numerical Solution of the Transcendental Equation using SOLVEX.BAS.

\begin{tabular}{llllll}
\hline $\boldsymbol{i}$ & $\boldsymbol{x}(\boldsymbol{i})$ & $\boldsymbol{f x}(\boldsymbol{i})$ & $\boldsymbol{i}$ & $\boldsymbol{x}(\boldsymbol{i})$ & $\boldsymbol{f} \boldsymbol{x}(\boldsymbol{i})$ \\
\hline 1 & 0.1 & $3.06 \mathrm{E}-01$ & 14 & 0.99812 & $1.77 \mathrm{E}-06$ \\
2 & 0 & $3.67 \mathrm{E}-01$ & 15 & 0.99884 & $6.75 \mathrm{E}-07$ \\
3 & 0.60003 & $7.03 \mathrm{E}-02$ & 16 & 0.99928 & $2.58 \mathrm{E}-07$ \\
4 & 0.74181 & $3.06 \mathrm{E}-02$ & 17 & 0.99956 & $9.85 \mathrm{E}-08$ \\
5 & 0.85131 & $1.05 \mathrm{E}-02$ & 18 & 0.99973 & $3.76 \mathrm{E}-08$ \\
6 & 0.90862 & $4.05 \mathrm{E}-03$ & 19 & 0.99983 & $1.44 \mathrm{E}-08$ \\
7 & 0.94447 & $1.51 \mathrm{E}-03$ & 20 & 0.9999 & $5.49 \mathrm{E}-09$ \\
8 & 0.96586 & $5.76 \mathrm{E}-04$ & 21 & 0.99994 & $2.10 \mathrm{E}-09$ \\
9 & 0.97901 & $2.19 \mathrm{E}-04$ & 22 & 0.99996 & $3.06 \mathrm{E}-10$ \\
10 & 0.98706 & $8.34 \mathrm{E}-05$ & 23 & 0.99998 & $1.17 \mathrm{E}-10$ \\
11 & 0.99201 & $3.18 \mathrm{E}-05$ & 24 & 0.99998 & $4.49 \mathrm{E}-11$ \\
12 & 0.99507 & $1.21 \mathrm{E}-05$ & 25 & 0.99999 & \\
13 & 0.99695 & $4.63 \mathrm{E}-06$ & & & \\
\hline
\end{tabular}

\section{Copyrights}

Copyright for this article is retained by the author(s), with first publication rights granted to the journal.

This is an open-access article distributed under the terms and conditions of the Creative Commons Attribution license (http://creativecommons.org/licenses/by/4.0/). 